\title{
Realistic Head Model Design and 3D Brain Imaging of NIRS Signals Using Audio Stimuli on Preterm Neonates for Intra-Ventricular Hemorrhage Diagnosis
}

\author{
Marc Fournier $^{1}$, Mahdi Mahmoudzadeh ${ }^{1}$, Kamran Kazemi ${ }^{2}$, Guy Kongolo ${ }^{1}$, \\ Ghislaine Dehaene-Lambertz ${ }^{3}$, Reinhard Grebe ${ }^{1}$, and Fabrice Wallois ${ }^{1}$ \\ ${ }^{1}$ GRAMFC, Inserm U1105, CHU Amiens, University of Picardie Jules Verne, Amiens, France \\ \{marc. fournier, mahdi.mahmoudzadeh, guy.kongolo, reinhard.grebe, \\ fabrice.wallois\}@u-picardie.fr \\ ${ }^{2}$ Dept. of Electrical and Electronics Engineering, Shiraz University of Technology, Shiraz, Iran \\ kazemi@sutech.ac.ir \\ ${ }^{3}$ NeuroSpin, CEA, Inserm U992, Cognitive Neuroimaging, University Paris XI, Paris, France \\ ghislaine.dehaene@cea.fr
}

\begin{abstract}
In this paper we propose an auditory stimulation and Near Infra-Red Spectroscopy (NIRS) hemodynamic changes acquisition protocol for preterm neonates. This study is designed to assess the specific characteristics of neurovascular coupling to auditory stimuli in healthy and ill neonate brains. The method could lead to clinical application in Intra-Ventricular Hemorrhage (IVH) diagnosis along with other techniques such as EEG. We propose a realistic head model creation with all useful head structures and brain tissues including the neonate fontanel for more accurate results from NIRS signals modeling. We also design a 3D imaging tool for dynamic mapping and analysis of brain activation onto the cortex surface. Results show significant differences in oxy-hemoglobin between healthy neonates and subjects with IVH.
\end{abstract}

Keywords: Head model design, 3D brain NIRS optical imaging, Hemodynamic response to audio stimuli, Neonate IVH diagnosis, Functional brain imaging.

\section{Introduction}

This paper introduces a Near Infra-Red Spectroscopy (NIRS) cerebral hemodynamic response optical monitoring method of the healthy and sick premature infant with IntraVentricular Hemorrhage (IVH). Non-invasive investigation of the oxygenation of the infant's brain is of high interest. The proposed method can be applied for early diagnosis of impairments in complement with other current brain imaging techniques such as ElectroEncephaloGraphy (EEG). Low arterial blood oxygenation and abnormal cerebral blood flow is believed to influence the function of the neonatal brain [1]. Preterm neonates are at high-risk of IVH because of their lack of ability to regulate cerebral blood flow and pressure [2]. Currently, brain injuries in infants are mainly diagnosed clinically by EEG (investigates neural function, but not vascular response) and cranial ultrasound (gives only anatomical information) [3]. Previous studies on cerebral hemodynamic 
responses using NIRS on neonates have been done using various stimulations such as visual [4] and auditory [5]. Visual stimuli in adults using NIRS has also been proposed while investigating head modeling and image reconstruction [6]. Another study on cerebral blood flow estimated by NIRS in preterm neonates has correlated NIRS findings with cerebral ultrasound results [7]. Children with delays in language and speech development are at high risk for later disorders in reading, spelling, and writing; academic skills which are highly dependent on language abilities. Brain injuries have been found to be associated with language and speech outcomes among children born prematurely [8]. IVH are risk factors for adver-se neurodevelopment outcomes, including cognitive impairment and cerebral palsy.

\section{$2 \quad$ Material and Methods}

\subsection{Subjects and NIRS Signals Acquisition Setup}

This study was carried out on two groups; the first one is composed of 12 healthy control subjects and the second one of 7 ill subjects with IVH of grade III-IV. All subjects of both groups are preterm neonates of gestational age from 28 to 32 weeks; tested during their sleep between 2 and 4 days after birth. Subjects were submitted to auditory stimuli which consist of two digitized syllables /ba/ and /ga/ as in a previous EEG study [9]. Three stimulations conditions were used: the standard one (ST: four /ba/ male); deviant voice (DV: three /ba/ male, one /ba/ female); and deviant phoneme (DP: three /ba/ male, one /ga/ male). The four syllables block duration is $4 \mathrm{~s}$ and total stimulation (20s) is composed of five consecutive blocks. A newborn special NIRS probe showed in Fig. 1(a) was designed and consists of two patches containing two detectors and sixteen light sources in each of them ( 8 to $\lambda=690 \mathrm{~nm} ; 8$ to $\lambda=830 \mathrm{~nm}$ wavelengths). Twenty acquisition channels, ten per hemisphere, are measured in the configuration showed in Fig. 1(b). We used a multi-channel frequency domain based optical imaging system (Imagent, ISS Inc.) for the acquisition of oxygenated hemoglobin $(\mathrm{HbO})$ and deoxygenated hemoglobin $(\mathrm{Hb})$ changes during auditory stimuli. Values of $\mathrm{HbO}$ and $\mathrm{Hb}$ and their changes were obtained using the relation between absorption spectroscopic coefficients of the environment and chromophore concentrations according to the modified Beer-Lambert law [10] used in NIRS studies.

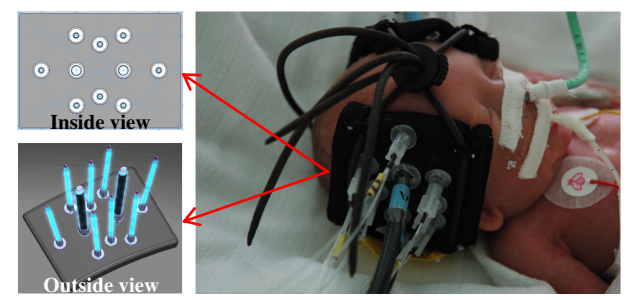

(a)

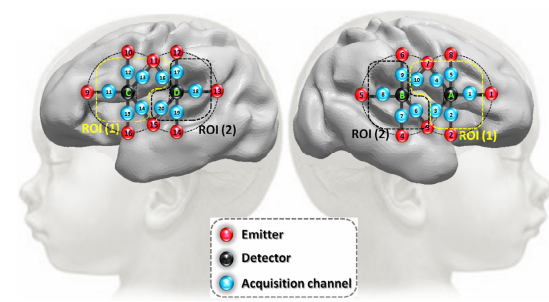

(b)

Fig. 1. NIRS acquisition probe design. (a) NIRS sensors patches design and its application to one subject. (b) Schematic positions and numbering of NIRS sensors according to both hemispheres with 4 detectors (A...D, black), 16 emitters (1...16, red) and 20 channels (1...20, blue). 


\subsection{Neonate Realistic Head Model Creation}

We created a 30 weeks (average of all healthy and ill subjects) preterm neonate complete head model with all useful head and brain structures used for NIRS signals mapping onto the brain. The first of three steps was a straightforward extraction from choosing the 30 weeks iteration of a previously created $4 \mathrm{D}(3 \mathrm{D}+\mathrm{t})$ preterm neonate MRI-based atlas [11]. This results in intracranial head model creation including cerebrospinal fluid, grey matter and white matter tissues. In the second step we created a full term neonate realistic head model from 15 subjects between 39 to 42 weeks of gestational age. MRI and CT-Scan of all subjects were acquired for clinical purposes and were found without any anatomical abnormalities after inspection. We used SPM toolbox (http://www.fil.ion.ucl.ac.uk/spm/) to perform co-registration of both imaging modalities, segmentation and space normalization of head and brain structures in order to build the atlas containing each structure probability mask. The fontanel was segmented from the CT-Scan using a variational level-set method [12]. The third step was achieved by registering the intracranial brain mask of the full term head model (created at step 2) to the intracranial brain mask of the preterm head model (created at step 1). The resulting transformation was then applied to the scalp, skull and fontanel of the full term model in order to fit these structures onto the preterm intracranial model; thus obtaining a complete preterm head model with all useful structures. Analysis of our full term neonate head model is resumed in Fig. 2 which shows the importance of considering the fontanel in neonate brain modeling; especially in temporal regions, of interest for this study, that are more widely covered by the fontanel.

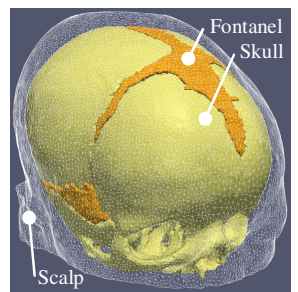

(a)

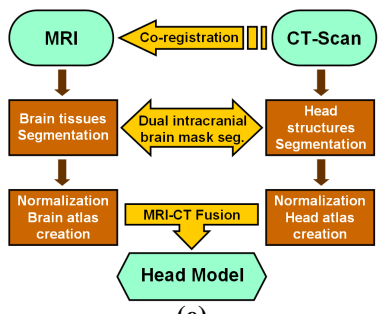

(e)

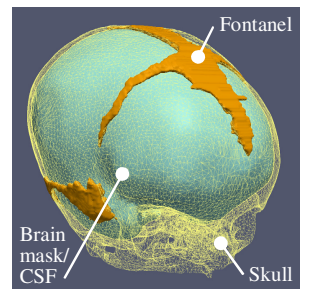

(b)

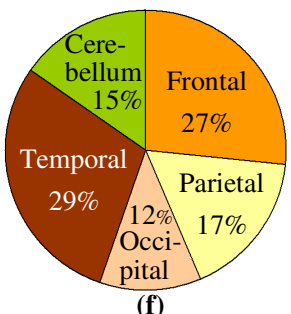

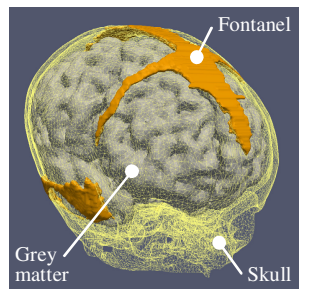

(c)

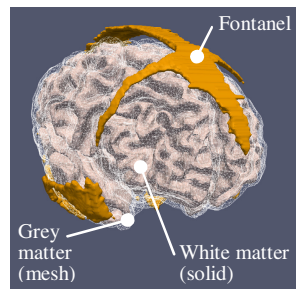

(d)

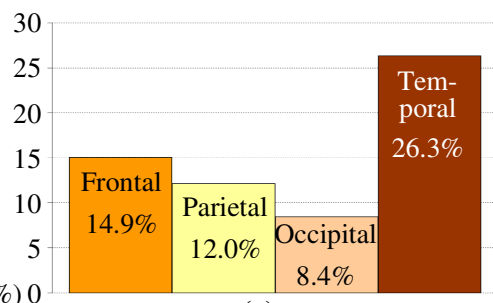

(g)

Fig. 2. Impact of fontanel in the head model. (a) to (d) Views of fontanel and other head and brain structures on one subject used in the head model creation. The brain is covered at $17 \%$ by the fontanel and $83 \%$ by the skull. (e) Procedure to create the full term head model (step two). (f) Proportion of the fontanel overlapping each brain lobes: this means $29 \%$ of the total fontanel surface overlaps both brain temporal lobes. (g) For each brain lobe, proportion of the overlapping fontanel compared to the total surface of the lobe: this means $26.3 \%$ of both temporal lobes surface is covered by the fontanel. Charts show average values for all subjects. 
To create our full term neonate head model we segment brain tissues from the MRI using the expectation maximization method [13]. To overcome the large grey levels overlap between different tissues in neonate MRI, we incorporate contextual and spatial a priori information during tissue classification using a mixture of Gaussians where each cluster is modeled by its mean, variance and a mixing proportion.

Skull and fontanel are segmented from CT-Scan. In neonates, the skull is composed of cranial bones and the fontanel. The cranial bones are obtained by applying automatic threshold to the image histogram and the fontanel can be identified as gaps between cranial bones of the skull. In order to extract the fontanel, a model-based variational level-set method is used to reconstruct the full skull; thus filling the fontanel gaps. Then the fontanel is obtained by removing cranial bones from the reconstructed full skull. Let $\varphi$ be the level-set function for segmentation and $\varphi_{m}$ be the signed distance function of the skull model. To reconstruct the full skull (including fontanel) the input image is segmented by minimizing the following functional:

$$
\begin{aligned}
& E\left(c_{1}, c_{2}, \varphi\right)=E_{C V}\left(c_{1}, c_{2}, \varphi\right)+E_{\text {shape }}(\varphi) \text {, with: } E_{\text {shape }}=\int_{\Omega}\left(\varphi(\vec{x})-\varphi_{m}(\vec{x})\right)^{2} d x \text {, and: } \\
& E_{C V}=\lambda_{1} \int_{\Omega}\left(I(\vec{x})-c_{1}\right)^{2} H(\varphi) d \vec{x}+\lambda_{2} \int_{\Omega}\left(I(\vec{x})-c_{2}\right)^{2}(1-H(\varphi)) d \vec{x}+v H(\varphi)+\mu|\nabla H(\varphi)|
\end{aligned}
$$

where $E_{C V}$ is a level-set formulation of the functional as previously proposed in [14] with $H(\varphi)=[1+(2 / \pi) \operatorname{atan}(\varphi / \varepsilon)] / 2$ being the heaviside function. $E_{\text {shape }}$ is used as shape prior to guide the segmentation as detailed in [12]; thus providing a constraint during evolution of the level-set function which is attracted to the skull.

Both MRI and CT atlases are created using the same method based on the intracranial brain mask $(B M)$ segmentation of each subject in order to register all other brain tissues and head structures $(S T)$ according to this procedure:

1. Select a reference subject: $B M_{R}$

2. Compute each subject affine transformation: $A_{i}=B M_{i} \rightarrow B M_{R}$

3. Normalize (first-pass) each subject structures: $S T_{i}{ }^{\prime}=A_{i} \times S T_{i}$

4. Compute cosine basis functions deformation fields: $D_{i}=B M_{i}{ }^{\prime} \rightarrow B M_{R}$

5. Normalize (second-pass) each subject structures: $S T_{i}{ }^{\prime \prime}=D_{i} \times S T_{i}{ }^{\prime}$

6. Compute nonlinear transf. of ref. subj. to all affine norm. subj.: $T_{i}=B M_{R} \rightarrow B M_{i}{ }^{\prime}$

7. Compute the mean transformation: $T_{M}=\operatorname{mean}\left(T_{i}\right)$

8. Compute spatially unbiased structures: $S T_{U i}=T_{M} \times S T_{i}{ }^{\prime \prime}$

9. Compute each structure average from individuals: $S T_{U A}=\operatorname{mean}\left(S T_{U i}\right)$

10. Smooth $S T_{U A}$ structures with a Gaussian kernel: $2 \mathrm{~mm}$ full-width half-maximum

To minimize the bias introduced by the chosen reference subject, this atlas creation process is repeated by replacing the initial reference intracranial target $\left(B M_{R}\right)$ with the first-pass intracranial model result. Finally the created MRI and CT atlases are fused together to create the multimodality neonatal atlas. The intracranial probabilistic model created from CT data is registered onto the MRI one. The obtained transformation parameters are applied to the CT atlas head structures in order to complete the MRI atlas brain tissues; thus obtaining our full term neonate head model. 


\subsection{Modeling and Imaging of NIRS Signals Propagation in Tissues}

At NIRS signals acquisition session, the position of each emitter and detector are digitized along with biomarkers reference positions. For each subject these 3D sensors positions are registered onto our average 30 weeks preterm neonate head model. Then the banana-shape photon path [15] through the head and brain tissues between each considered pair of emitter/detector are computed. Fig. 3(a) shows a schematic of the photon path between a pair of emitter/detector. Fig. 3(b) shows one subject example of the 3D head model with the sensors positions and the computed photon paths. The photons migration along the path between the emitter and detector is modeled by a probability distribution [16] which occurs with a hitting density expressed by:

$$
\begin{gathered}
P(x, y, z)=\frac{z^{2} \exp [-k(\sqrt{A}+\sqrt{B})] \cdot(k \sqrt{A}+1) \cdot(k \sqrt{B}+1)}{A^{3 / 2} \cdot B^{3 / 2}} \\
\text { with }: A=x^{2}+y^{2}+z^{2}, B=(d-x)^{2}+y^{2}+z^{2}, \text { and }: k=\sqrt{3 \mu_{a} \mu_{s}}
\end{gathered}
$$

where $d$ is the emitter/detector distance $(15 \mathrm{~mm}), \mu_{a}$ the absorption coefficient and $\mu_{s}$ the reduced scattering coefficient of the environment. Our anatomical head model is used to determine to which tissue belongs each voxel in order to locally compute the suitable $k$ based on the different values of $\mu_{a}$ and $\mu_{s}$ provided in [17] for each tissue. It has been demonstrated in [17] that considering the fontanel tissue in our head model leads to a more realistic modeling from NIRS signals. For each subject we constraint equation (2) to the grey matter of the preterm head model which is filled with information according to all photon paths and their distributions. Each pair-wise sensor photon path may partially overlap into some voxels; resulting in multiple values for a single voxel. Thus an average function is used to output a final value per voxel. Then a grand average is performed over all subjects and brain activity is normalized. For visualization purpose, brain activations in the grey matter volume are integrated to the cortex surface by orthogonal projection onto the brain folds themselves. This procedure is repeated for each time steps of the recorded NIRS signals. All generated 3D images of brain activations build a real time series of dynamic topography mapping.

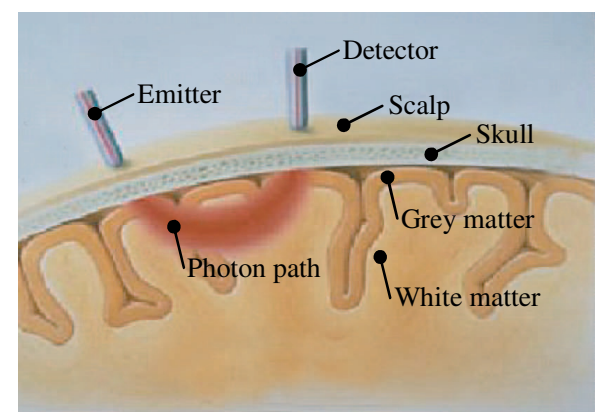

(a)

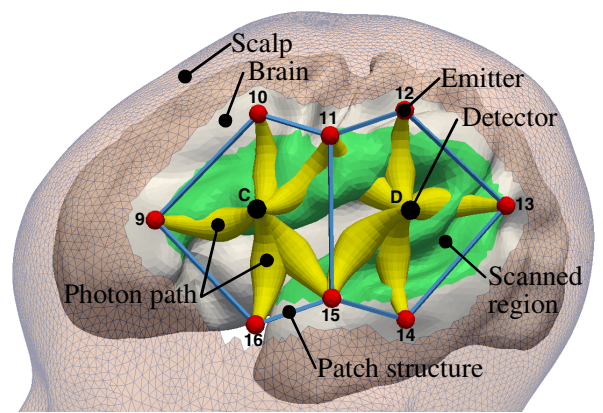

(b)

Fig. 3. Photon path and its 3D modeling. (a) Schematic of the banana-shape photon path trough head tissues between sensors. (b) Left hemisphere view of the 3D head model (only scalp and brain are showed) with NIRS sensors registered and 3D photon paths trough the grey matter. 


\section{$3 \quad$ Results}

NIRS signals are acquired at a frequency of $9.2 \mathrm{~Hz}$ along with oxygen saturation and cardio-respiratory sensors data used in preprocessing to reject artifacts due to systemic and non-cerebral modifications. A software application has been designed to compare in real time healthy and IVH subjects dynamic brain activations images generated as described in previous section 2.3. This tool allows analysis of dynamic mapping of NIRS signals onto the brain surface which runs from $-5 \mathrm{~s}$ ( $5 \mathrm{~s}$ before stimuli start, used for baseline information) to $+25 \mathrm{~s}$ ( $5 \mathrm{~s}$ after the $20 \mathrm{~s}$ of stimuli, used for resting state).

Fig. 4(a) is an average image of 5s activity of NIRS HbO signals. It was computed using the dynamic real time brain activation mapping application. The average period from $5 \mathrm{~s}$ to $10 \mathrm{~s}$ of stimulation time was chosen for its high and peak activity as shown in most $\mathrm{HbO}$ channels in Fig. 4(b). For each stimulation condition (ST, DV, DP) taken individually and for all conditions together, Fig. 4(a) shows higher brain activation in normal subjects compared to abnormal IVH ones.

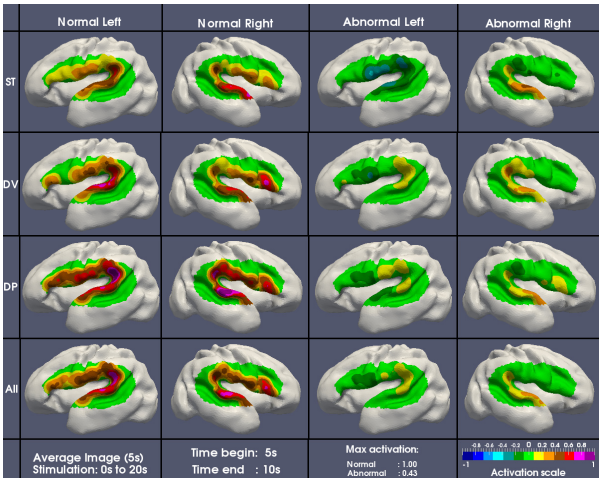

(a)
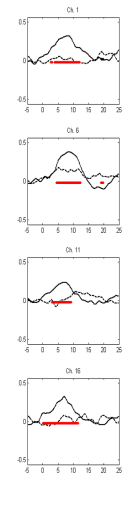

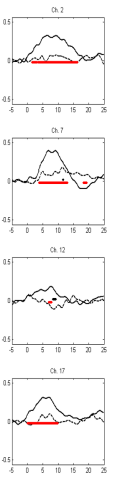

.

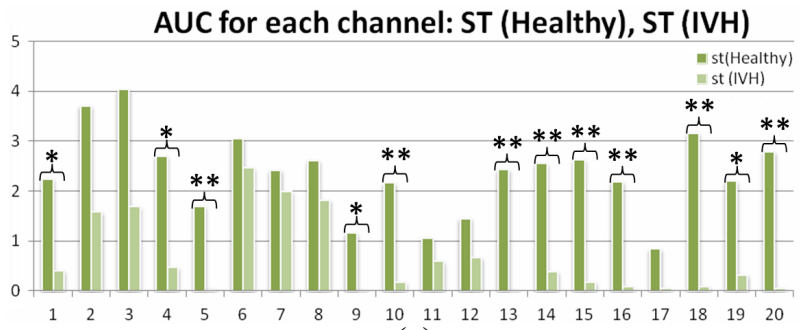

(c)

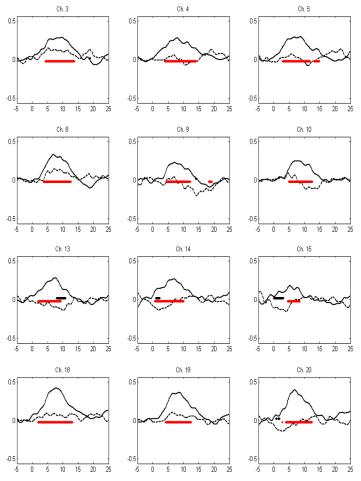

(b)

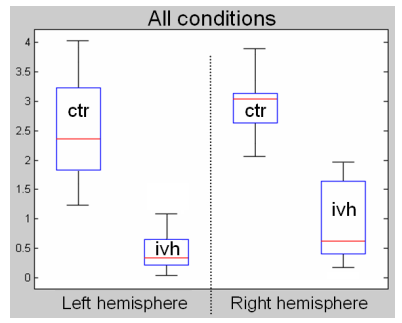

(d)

Fig. 4. Images and signals comparisons between healthy and IVH subjects. (a) Average image of $5 \mathrm{~s}$ ( $5 \mathrm{~s}$ to $10 \mathrm{~s}$ ) showing much more activation in healthy subjects for each condition. (b) Grand average $\mathrm{HbO}$ signals for the 20 channels over all conditions and significant part of the signals at $\mathrm{p}<0.05$ corrected for multiple comparisons. (c) AUC per channel for ST condition. Channels marked with at least one star $(*)$ show significant t-test difference at $\mathrm{p}<0.05$. Channels marked with two stars (**) are corrected for multiple comparisons. (d) AUC per hemisphere for all conditions showing no overlap between healthy control (ctr) and IVH subjects. 
The strongest brain activation shown in Fig. 4(a) for IVH is only $43 \%$ of the maximum in normal subjects and it is only visible on the planum temporal of right hemisphere for standard condition. Otherwise the maximum activation of IVH found elsewhere is around $25 \%$ of healthy subjects. Normal control subjects show high activation in all conditions and most regions of interest. Fig. 4(b) shows the 20 NIRS channels $\mathrm{HbO}$ signals of all conditions averaged together. The solid line is for healthy subjects and the doted line for IVH ones. The gap between the levels of activation of both groups is obvious in all channels. The horizontal red (healthy) and black (IVH) bars show the significant part of the signals compared to the baseline ( $-5 \mathrm{~s}$ to $0 \mathrm{~s})$ at $\mathrm{p}<0.05$ corrected for multiple comparisons using Holm's method for small samples [18] implemented in FieldTrip toolbox (http://fieldtrip.fcdonders.nl/). The maximum number of permutations for each group has been used. This result shows large significant activations in most channels for healthy subjects and almost no significant activation for IVH subjects. The main significant part of IVH signals are almost exclusively desactivation (negative values) which reinforce the gap between both groups.

Fig. 4(c) shows the area under curve (AUC) integration values for each channel signals in the standard condition for both groups. Standard condition has been selected because it shows less activation in the healthy group. Moreover the AUC was computed for the positive part of the signals only since IVH subjects show more desactivation. These choices have been made to reduce to minimum the gap between both groups. Even in these analysis conditions, Fig. 4(c) result shows statistically significant differences between both groups in the majority of the channels which are star marked (* t-test at $\mathrm{p}<0.05 ; * *$ corrected with Holms method). Fig. 4(d) shows another AUC result for all conditions together and per hemisphere which means average values for the ten channels on each side have been used. This box-plot type result shows no overlap at all between both groups in AUC of all individual subjects; suggesting a strong significant difference between both groups.

\section{Conclusion}

The present data confirm the existence of neurovascular coupling in premature brain, although it may be impaired in ill subjects. It also shows that ill premature neonates are unable to process syllabic stimuli, a step for language acquisition ability. This inability of pathological brain to adapt to either endogenous or exogenous stimuli by an increase in blood flow can represent a mechanism by which the pathological brain enters in a deleterious pathological loop. This might explain cerebral disabilities observed latter in acquisitions throughout the neurodevelopment [8]. Our audio stimuli and NIRS acquisition protocol coupled to the designed head model and 3D dynamic mapping of brain activation could represent a valuable tool for IVH diagnosis and for a wider range of applications as well. On top of the binary classification of a subject (healthy or IVH), the proposed imaging solution enables: i) a multimodality image-based analysis to compare and complete other sources information such as EEG, ultrasound scan and fMRI; and ii) a shape/functional related local region analysis such as their asymmetries. 


\section{References}

1. Volpe, J.J.: Brain injury in the premature infant: Neuropathology, clinical aspects, and pathogenesis. Clin. Perinatol. 3, 567-587 (1997)

2. Ballabh, P.: Intraventricular hemorrhage in premature infants: mechanism of disease. Pediatr. Res. 67, 1-8 (2010)

3. Gibson, A.P., Hebden, J.C., Arridge, S.R.: Recent advances in diffuse optical imaging. Phys. Med. Biol. 50, 1-43 (2005)

4. Hoshi, Y., Kohri, S., Matsumoto, Y., Cho, K., Matsuda, T., Okajima, S., Fujimoto, S.: Hemodynamic responses to photic stimulation in neonates. Pediatr. Neurol. 23, 323-327 (2000)

5. Sakatani, K., Chen, S., Lichty, W., Zuo, H., Wang, Y.P.: Cerebral blood oxygenation changes induced by auditory stimulation in newborn infants measured by near infrared spectroscopy. Early Hum. Dev. 55, 229-236 (1999)

6. Dehghani, H., White, B.R., Zeff, B.W., Tizzard, A., Culver, J.P.: Depth sensitivity and image reconstruction analysis of dense imaging arrays for mapping brain function with diffuse optical tomography. Applied Optics 48, D137-D143 (2009)

7. Meek, J.H., Tyszczuk, L., Elwell, C.E., Wyatt, J.S.: Low cerebral blood flow is a risk factor for severe intraventricular haemorrhage. Arch. Dis. Child Fetal Neonatal Ed. 81, 15-18 (1999)

8. Downie, A.L., Frisk, V., Jakobson, L.S.: The impact of periventricular brain injury on reading and spelling abilities in the late elementary and adolescent years. Child Neuropsychol. 11, 479-495 (2005)

9. Dehaene-Lambertz, G.: Cerebral specialization for speech and non-speech stimuli in infants. J. Cogn. Neurosci. 12, 449-460 (2000)

10. Delpy, D.T., Cope, M., Van-Der-Zee, P., Arridge, S., Wray, S., Wyatt, J.: Estimation of optical pathlength through tissue from direct time of flight measurement. Phys. Med. Biol. 33, 1433-1442 (1988)

11. Murgasova, M., Aljabar, P., Srinivasan, L., Counsell, S.J., Doria, V., Serag, A., Gousias, I.S., Boardman, J.P., Rutherford, M.A., Edwards, A.D., Hajnal, J.V., Rueckert, D.: A dynamic 4D probabilistic atlas of the developing brain. Neuroimage 54, 2750-2763 (2011)

12. Jafarian, N., Kazemi, K., Abrishami-Moghaddam, H., Grebe, R., Fournier, M., Helfroush, M.S., Gondry-Jouet, C., Wallois, F.: Automatic segmentation of newborns' skull and fontanel from CT data using model-based variational level set. Signal Image and Video Processing (to appear, 2012), doi:10.1007/s11760-012-0300-x

13. Ashburner, J., Friston, K.J.: Unified segmentation. Neuroimage 26, 839-851 (2005)

14. Chan, T.F., Vese, L.A.: Active contours without edges. IEEE Trans. Image Process. 10, 266-277 (2001)

15. Bunce, S., Izzetoglu, M., Izzetoglu, K., Onaral, B., Pourrezaei, K.: Functional nearinfrared spectroscopy: an emerging neuro-imaging modality. Eng. Med. Biol. 25, 54-62 (2006)

16. Sassaroli, A., Frederick, B., Tong, Y., Renshaw, P.F., Fantini, S.: Spatially weighted BOLD signal for comparison of functional magnetic resonance imaging and near-infrared imaging of the brain. Neuroimage 33, 505-514 (2006)

17. Dehaes, M., Kazemi, K., Pelegrini-Issac, M., Grebe, R., Benali, H., Wallois, F.: Quantitative effect of the neonatal fontanel on synthetic near infrared spectroscopy measurements. Hum. Brain Mapp. (2011) (to appear), doi:10.1002/hbm.21483

18. Holm, S.: A simple sequentially rejective multiple test procedure. Scand. J. Statist. 6, 65-70 (1979) 